\title{
NÍVEL DE CONHECIMENTO DOS PROFISSIONAIS DA ESTRATÉGIA DE SAÚDE DA FAMÍLIA SOBRE A RELAÇÃO BIDIRECIONAL DOENÇA PERIODONTA - DIABETES MELLITUS
}

\author{
LEVEL OF KNOWLEDGE OF FAMILY HEALTH STRATEGY'S \\ PROFESSIONALS REGARDING THE PERIODONTAL DISEASE - \\ DIABETES MELLITUS BIDIRECTIONAL RELATIONSHIP
}

\author{
Danielly Vieira Gomes ${ }^{1}$, Eduardo Sérgio Donato Duarte Filho², Renata de Oliveira Cartaxo³, \\ João Braga da Silva Junior ${ }^{1}$, Anna Karolline Cadengue de Siqueira ${ }^{1}$ \\ 1. Graduados em Odontologia pela Universidade de Pernambuco - UPE. \\ 2. Doutor em Odontologia. Professor Adjunto de Odontologia da UPE e Professor de Odontologia do Centro Universitário Tabosa de Almeida - \\ ASCES-UNITA. \\ 3. Doutoranda em Ciências da Saúde. Professora Assistente de Odontologia da Universidade de Pernambuco - UPE.
}

\section{Palavras-chave:}

Diabetes Mellitus; Doenças Periodontais, Conhecimentos, Atitudes e Práticas em Saúde.

\begin{abstract}
RESUMO
A relação entre as condições de saúde sistêmica e doença periodontal é bidirecional, na qual a manutenção de uma auxilia no controle da outra. O diabetes é uma dessas condições, que pode aumentar a gravidade e extensão da doença periodontal, e em contramão, a infecção periodontal pode agravar o controle glicêmico. Objetiva-se avaliar o nível de conhecimento dos profissionais atuantes na Equipe de Saúde da Família (ESF), em Arcoverde/PE, em relação à doença periodontal e o Diabetes mellitus. O estudo é de caráter quantitativo, exploratório e transversal, no qual foi aplicado um questionário adaptado da literatura com 18 perguntas prevalentemente objetivas a 38 profissionais de saúde (médicos, enfermeiros e cirurgiões dentistas) atuantes em 13 Unidades Básicas de Saúde. Constatou-se que a maioria dos profissionais participantes apresentam bom nível de conhecimento acerca dos principais exames laboratoriais empregados para o diagnóstico do diabetes. Entretanto, no que se refere à influência que o tratamento periodontal básico oferece sobre o controle glicêmico dos pacientes diabéticos descompensados, $50 \%$ dos médicos, $62 \%$ dos enfermeiros e $31 \%$ dos dentistas não conheciam tal mecanismo. Pode-se afirmar que o conhecimento sobre a relação Diabetes mellitus e doença periodontal ainda é escasso entre expressiva parte dos profissionais.
\end{abstract}

\section{ABSTRACT}

The relationship between the conditions of systemic health and periodontal disease is two-way, in which the maintenance of one helps in the control of the other. Diabetes is one such condition, which can increase the severity and extension of periodontal disease, and on the other hand, periodontal infection may aggravate glycemic control. The aim is to evaluate the knowledge of the professionals working in the "Family Health Strategy's", in Arcoverde / PE, in relation to periodontal disease and Diabetes mellitus. Quantitative, exploratory and cross-sectional study, in which a questionnaire adapted from the literature was applied with 18 predominantly objective questions to 38 health professionals (doctors, nurses and dentists) working in 13 Basic Health Units. it was verified that the majority of the participants present a good level of knowledge about the main laboratory tests used for the diagnosis of diabetes. However, regarding the influence that basic periodontal treatment has on glycemic control of patients decompensated diabetics, $50 \%$ of doctors, $62 \%$ of nurses and $31 \%$ of dentists did not know such mechanism. It can be affirmed that the knowledge about the relation diabetes and periodontal disease is still scarce among expressive part of the professionals.
Autor Correspondente:

Eduardo Sérgio Donato Duarte Filho

R. Arlindo Porto, 220, ap 1102 - Maurício de Nassau

Caruaru - PE, CEP: 55014-265, e-mail: eduardo.sergio@upe.br

\section{INTRODUÇÃO}

A doença periodontal (DP) representa um grave problema de saúde pública odontológica, tanto nos países subdesenvolvidos quanto nos desenvolvidos ${ }^{1}$. No Brasil, a DP é um dos principais agravos que acometem a saúde bucal e é objeto de estudos epidemiológicos em virtude de sua elevada prevalência e gravidade ${ }^{2,3}$, sendo uma das principais causas de perda dentária em adultos ${ }^{4,5}$.

As DPs que mais acometem a população são a gengivite, que se desenvolve em poucos dias e caracteriza-se por mudanças inflamatórias e se limitam ao tecido gengival, 
induzidas pelo acúmulo do biofilme supra gengival sobre superfície dentária ${ }^{1,2}$, e a periodontite, definida como uma doença inflamatória dos tecidos de suporte dos dentes, é causada por microrganismos específicos, que resultam em uma destruição progressiva do ligamento periodontal e osso alveolar, com formação de bolsa, retração ou ambas ${ }^{6,7}$.

A relação entre as condições de saúde sistêmica e a doença periodontal é bidirecional, na qual a manutenção de uma auxilia no controle da outra ${ }^{8}$. O diabetes é uma condição sistêmica que pode aumentar a gravidade e extensão da $\mathrm{DP}^{6,9}$, alterando, por exemplo, o curso da periodontite ${ }^{10}$. Colocar a DP como uma complicação do diabetes, se justifica pelo fato de existir maiores prevalência e gravidade da periodontite em pacientes diabéticos ${ }^{8}$.

O Diabetes mellitus (DM) é uma desordem metabólica de etiologia multifatorial, com alta prevalência, que constitui um sério problema de saúde pública global' Mundialmente, estima-se que cerca de 285 milhões indivíduos são afetados ${ }^{11}$.

A hiperglicemia provocada pelo DM ocasiona modificações nas funções de células do sistema imune, além de tecidos e órgãos, reduzindo colágeno, alterando fatores de crescimento, aumentando apoptose e estresse oxidativo, diminuindo matriz extracelular, desregulando citocinas e alterando o fluxo salivar, resultando nas alterações periodontais $^{12}$. A hiperresponsividade para antígenos bacterianos seria a via para uma elevada produção de citocinas e mediadores inflamatórios, resultando numa inflamação intensa, que pode repercutir de maneira significativa na destruição periodontal ${ }^{1}$. Assim, pacientes com diabetes têm maior probabilidade de desenvolver DP7,11.

A DP é um fator de risco para o controle glicêmico em pacientes descompensados, tendo em vista que a inflamação, de maneira geral, induz resistência insulínica, não diferentemente do quadro inflamatório da DP, já que ela pode também aumentar o estado inflamatório sistêmico ${ }^{11}$. Nessa perspectiva, o tratamento periodontal tem sucesso limitado na presença de diabetes não diagnosticado ou mal controlado. Em contramão, a infecção periodontal pode agravar o controle glicêmico, e deve ser tratada de forma urgente ${ }^{6}$.

A Estratégia de Saúde da Família (ESF) organizase a partir de uma equipe multiprofissional (composta em sua formação básica por médico, enfermeiro, agentes comunitários de saúde e auxiliar ou técnico de enfermagem havendo oferta de saúde bucal na equipe, o cirurgião dentista e seu auxiliar passam a integrar a equipe). Entretanto, nem sempre esses profissionais estão preparados e com disposição para atuar de forma integrada. Dessa maneira, é primordial a integração dos profissionais de saúde sobre esta relação bidirecional entre o diabetes e DP, sendo imprescindível o tratamento interdisciplinar para que o paciente seja orientado de forma adequada sobre os cuidados necessários com a saúde periodontal e sistêmica ${ }^{8}$.

Em vista do exposto, o presente trabalho objetivou avaliar o conhecimento dos profissionais atuantes na Equipe de Saúde da Família, em Arcoverde/PE, no que se refere à relação bidirecional entre a doença periodontal e o Diabetes mellitus.

\section{MATERIAL E MÉTODOS}

O estudo é transversal, exploratório, de campo e de caráter quantitativo. Foi realizado nas Unidades de Saúde da Família (USFs) da cidade de Arcoverde/PE, com profissionais de nível superior das equipes.

O recorte do cenário de pesquisa se deu por conveniência, nos diferentes bairros do setor urbano da cidade. Dentre as 24 USFs do município, foram selecionadas 13 $(50 \%+1)$ conforme ilustra a Figura 1.

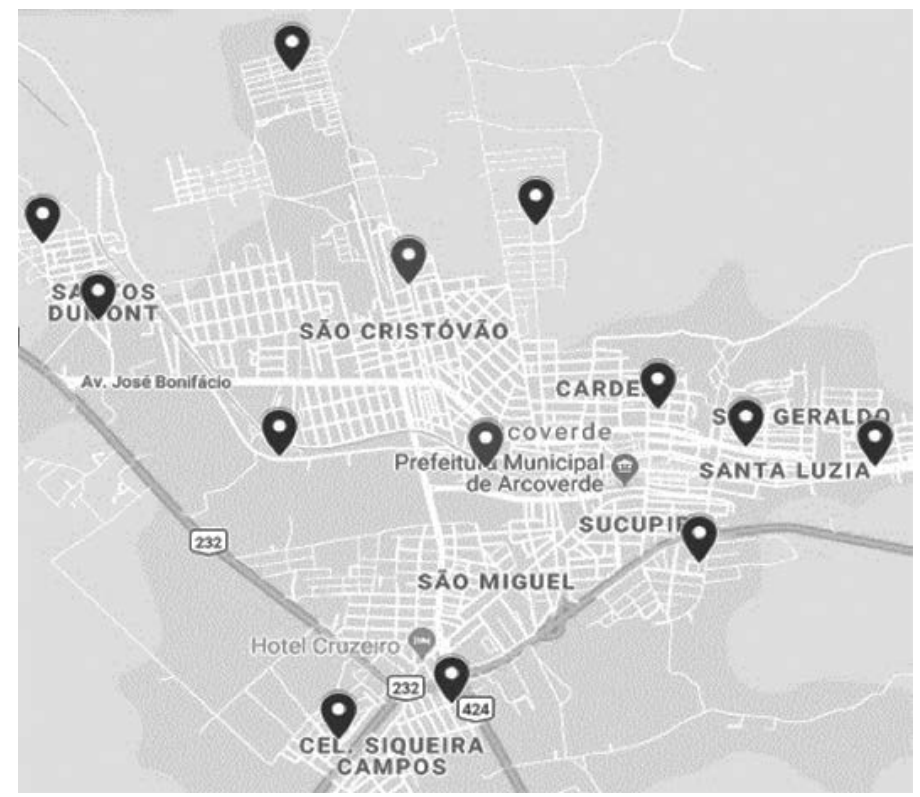

Fonte: Elaboração própria utilizando a ferramenta do Google Maps.

Figura 1 - Mapa territorial das UBSFs abordadas em Arcoverde/PE. 
Definido o campo de pesquisa, foram convidados a participar da pesquisa os médicos, enfermeiros e cirurgiõesdentistas (CDs) atuantes das USFs selecionadas. Foram utilizados dois tipos de Questionários com 18 perguntas cada, dos quais o Tipo 1 era destinado aos Médicos e Enfermeiros, e o Tipo 2 aos Cirurgiões-Dentistas. A diferença básica entre os dois tipos de questionários se deu apenas à questão 18 , pois se tratou de uma pergunta voltada para o profissional de acordo com sua conduta (Figura 2). Estes questionários foram elaborados com base no estudo de Santos e colaboradores ${ }^{13}$, que possuía objetivos semelhantes ao presente estudo.

Os questionários foram aplicados nas respectivas USFs de atuação de cada um dos profissionais, durante o horário de trabalho mais conveniente para o profissional em questão. O período de coleta ocorreu entre os meses de setembro de 2018 a março de 2019 e foi realizada uma análise descritiva utilizando o programa Microsoft Excel ${ }^{\circledR}$ versão 2013.

A pesquisa foi autorizada para sua execução após aprovação pelo Comitê de Ética em Pesquisa da Universidade de Pernambuco, sob o parecer $n^{\circ} 2.808 .876$ e, em respeito à resolução 466/12 do Conselho Nacional de Saúde, os profissionais participantes assinaram o Termo de Consentimento Livre e Esclarecido concordando em participar do estudo, para que em seguida respondessem ao questionário.

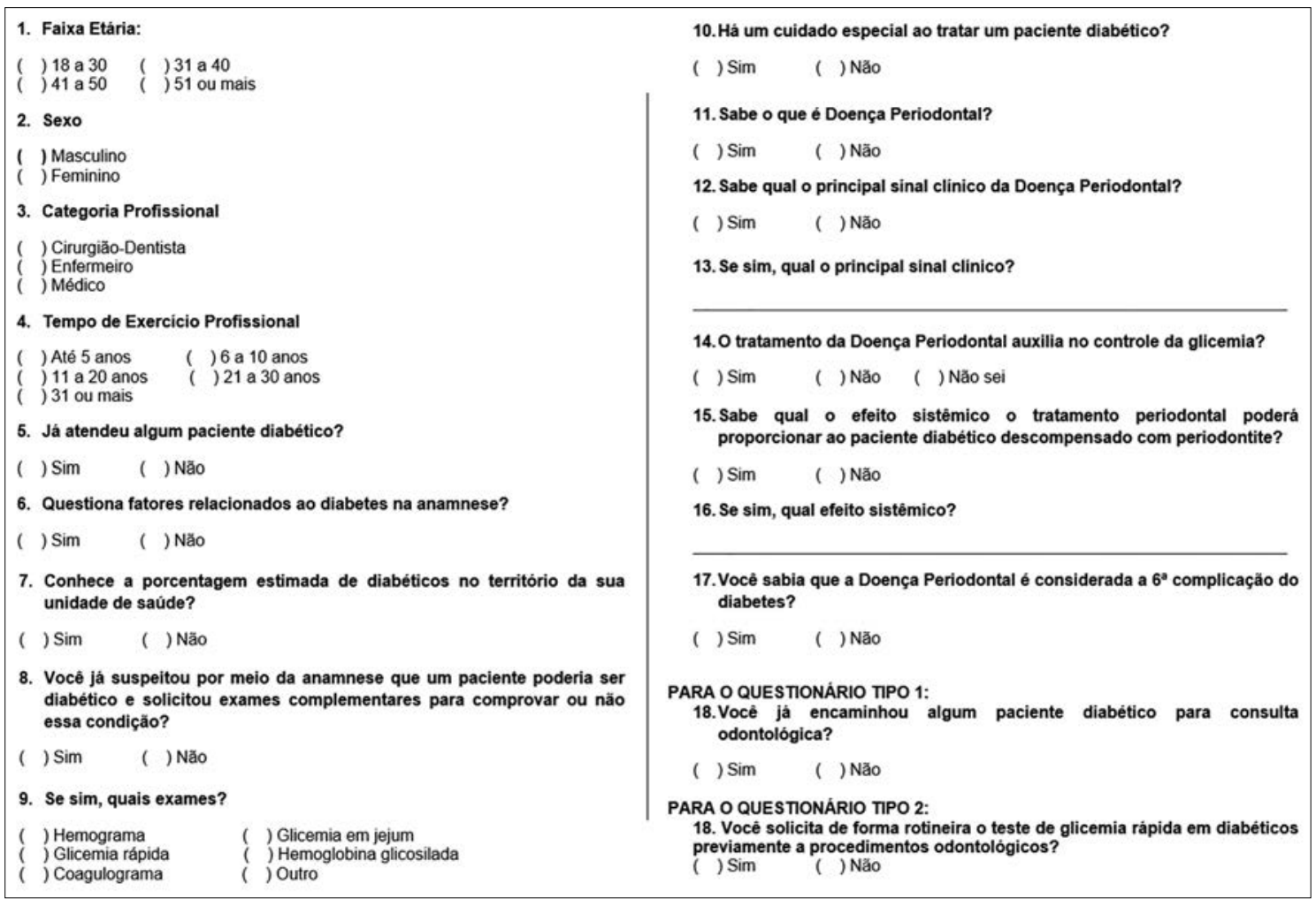

Figura 2 - Questionário de Pesquisa (compilados os “Tipos 1"e“2").

\section{RESULTADOS}

Das treze USFs selecionadas, participaram da pesquisa 12 Médicos (1 médico se negou a participar), 13 Cirurgiões-Dentistas e 13 Enfermeiros, totalizando 38 profissionais. Destes, $26 \%$ pertenciam a faixa etária (em anos) de 18 a 30,50\% de 31 a 40,13\% de 41 a 50 e 10\% com 51 anos ou mais. Quanto ao sexo, 47\% dos participantes são masculinos e 53\% femininos. Em relação ao tempo de atuação profissional, 23\% dos participantes têm até 5 anos de atuação; $29 \%$ possuem de 6 a 10 anos de atividade profissional; 31\% já trabalham de 11 a 20 anos, enquanto 5\% estão em atividade de 21 a 30 anos e, 10\% relataram apresentar 31 ou mais anos atuação profissional.

A frequência dos dados referentes ao conhecimento dos profissionais sobre DM está representada na Tabela 1. Já os resultados do conhecimento sobre a DP, DM e a relação entre as patologias estão expressos na Tabela 2. 
Conhecimento profissional na ESF: DP-DM

Gomes DV, et al.

Tabela 1 - Frequência dos dados sobre o conhecimento de Diabetes mellitus entre os profissionais.

\begin{tabular}{|c|c|c|c|c|c|c|c|c|}
\hline \multirow{3}{*}{ Variável } & \multicolumn{8}{|c|}{ Categoria Profissional } \\
\hline & \multicolumn{2}{|c|}{ Médico } & \multicolumn{2}{|c|}{ CD } & \multicolumn{2}{|c|}{ Enfermeiro } & \multicolumn{2}{|c|}{ Média } \\
\hline & $\mathbf{N}$ & $\%$ & $\mathbf{N}$ & $\%$ & $\mathbf{N}$ & $\%$ & $\mathbf{N}$ & $\%$ \\
\hline \multicolumn{9}{|c|}{ Já atendeu algum paciente diabético? } \\
\hline Sim & 12 & 100 & 13 & 100 & 13 & 100 & 38 & 100 \\
\hline Não & - & - & - & - & - & - & - & - \\
\hline Total & 12 & 100 & 13 & 100 & 13 & 100 & 38 & 100 \\
\hline \multicolumn{9}{|c|}{ Questiona fatores relacionados ao diabetes na anamnese? } \\
\hline Sim & 12 & 100 & 13 & 100 & 13 & 100 & 38 & 100 \\
\hline Não & - & - & - & - & - & - & - & - \\
\hline Total & 12 & 100 & 13 & 100 & 13 & 100 & 38 & 100 \\
\hline \multicolumn{9}{|c|}{ Conhece a porcentagem estimada de diabéticos no território de sua unidade de saúde? } \\
\hline Sim & 7 & 58,33 & 6 & 46,15 & 13 & 100 & 26 & 68,42 \\
\hline Não & 5 & 41,66 & 7 & 53,84 & - & - & 12 & 31,57 \\
\hline Total & 12 & 100 & 13 & 100 & 13 & 100 & 38 & 100 \\
\hline \multicolumn{9}{|c|}{$\begin{array}{l}\text { Você já suspeitou por meio da anamnese que um paciente poderia ser diabético e solicitou exames complementares } \\
\text { para comprovar ou não essa condição? }\end{array}$} \\
\hline Sim & 12 & 100 & 11 & 84,61 & 13 & 100 & 36 & 94,76 \\
\hline Não & - & - & 2 & 15,38 & - & - & 2 & 5,26 \\
\hline Total & 12 & 100 & 13 & 100 & 13 & 100 & 38 & 100 \\
\hline \multicolumn{9}{|l|}{ Se sim, quais exames? } \\
\hline Hemograma & 5 & 41,66 & 2 & 18,18 & 2 & 15,38 & 8 & 22,22 \\
\hline Glicemia rápida & 7 & 58,33 & 6 & 54,54 & 8 & 61,53 & 21 & 55,26 \\
\hline Coagulograma & 1 & 8,33 & 2 & 18,18 & - & - & 3 & 7,89 \\
\hline Glicemia em Jejum & 11 & 91,66 & 10 & 90,90 & 11 & 84,61 & 32 & 84,21 \\
\hline Hemoglobina glicosilada & 11 & 91,66 & 4 & 36,36 & 9 & 69,23 & 24 & 63,15 \\
\hline Outro & 2 & 16,66 & - & - & 6 & 46,15 & 8 & 22,05 \\
\hline Total & 12 & 100 & 11 & 100 & 13 & 100 & 38 & 100 \\
\hline \multicolumn{9}{|c|}{ Há um cuidado especial ao tratar um paciente diabético? } \\
\hline Sim & 11 & 91,66 & 13 & 100 & 12 & 92,30 & 36 & 94,73 \\
\hline Não & 1 & 8,33 & - & - & 1 & 7,69 & 2 & 5,26 \\
\hline Total & 12 & 100 & 13 & 100 & 13 & 100 & 38 & 100 \\
\hline
\end{tabular}

Tabela 2 - Dados sobre o conhecimento entre DM, DP e, sua relação bidirecional.

\begin{tabular}{lcccccccc}
\hline & \multicolumn{7}{c}{ Categoria Profissional } \\
\cline { 2 - 10 } & Variável & $\mathbf{2}$ Médico & \multicolumn{1}{c}{ CD } & \multicolumn{2}{c}{ Enfermeiro } & \multicolumn{2}{c}{ Total } \\
\cline { 2 - 10 } & $\mathbf{N}$ & $\%$ & $\mathbf{N}$ & $\%$ & $\mathbf{N}$ & $\%$ & $\mathbf{N}$ & $\%$ \\
\hline Sabe o que é doença periodontal (DP)? & & & & & & & \\
Sim & 8 & 66,66 & 13 & 100 & 5 & 38,46 & 26 & 68,42 \\
Não & 4 & 33,33 & - & - & 8 & 61,53 & 12 & 31,57 \\
\hline Total & $\mathbf{1 2}$ & $\mathbf{1 0 0}$ & $\mathbf{1 3}$ & $\mathbf{1 0 0}$ & $\mathbf{1 3}$ & $\mathbf{1 0 0}$ & $\mathbf{3 8}$ & $\mathbf{1 0 0}$ \\
\hline & & & & & & & & continua...
\end{tabular}


Tabela 2 - Continuação

\begin{tabular}{|c|c|c|c|c|c|c|c|c|}
\hline \multirow{3}{*}{ Variável } & \multicolumn{8}{|c|}{ Categoria Profissional } \\
\hline & \multicolumn{2}{|c|}{ Médico } & \multicolumn{2}{|c|}{ CD } & \multicolumn{2}{|c|}{ Enfermeiro } & \multicolumn{2}{|c|}{ Total } \\
\hline & $\mathbf{N}$ & $\%$ & $\mathbf{N}$ & $\%$ & $\mathbf{N}$ & $\%$ & $\mathbf{N}$ & $\%$ \\
\hline \multicolumn{9}{|c|}{ Sabe qual o principal sinal clínico da doença periodontal (DP)? } \\
\hline Sim & 5 & 41,66 & 13 & 100 & 3 & 23,07 & 21 & 55,26 \\
\hline Não & 7 & 58,33 & - & - & 10 & 76,92 & 17 & 44,73 \\
\hline Total & 12 & 100 & 13 & 100 & 13 & 100 & 38 & 100 \\
\hline \multicolumn{9}{|c|}{ Se sim, qual o principal sinal clínico? } \\
\hline Sangramento & 3 & 25 & 6 & 46,15 & 2 & 15,38 & 11 & 28,94 \\
\hline Bolsa Periodontal & - & - & 5 & 38,46 & - & - & 5 & 13,15 \\
\hline Outro & 2 & 16,66 & 2 & 15,38 & 1 & 7,69 & 5 & 13,15 \\
\hline Não respondeu & 7 & 58,33 & - & - & 10 & 76,92 & 17 & 44,73 \\
\hline Total & 12 & 100 & 13 & 100 & 13 & 100 & 38 & 100 \\
\hline \multicolumn{9}{|c|}{ O tratamento da doença periodontal auxilia no controle da glicemia? } \\
\hline $\operatorname{Sim}$ & 7 & 58,33 & 10 & 76,92 & 6 & 46,15 & 23 & 60,52 \\
\hline Não & - & - & 1 & 7,69 & - & - & 1 & 2,63 \\
\hline Não sei & 5 & 41,66 & 2 & 15,38 & 7 & 53,84 & 14 & 36,84 \\
\hline Total & 12 & 100 & 13 & 100 & 13 & 100 & 38 & 100 \\
\hline
\end{tabular}

Sabe qual o efeito sistêmico o tratamento periodontal poderá proporcionar ao paciente diabético descompensado com periodontite?

\begin{tabular}{lcccccccc} 
Sim & 6 & 50 & 9 & 69,23 & 5 & 38,46 & 20 & 52,63 \\
Não & 6 & 50 & 4 & 30,76 & 8 & 61,53 & 18 & 47,36 \\
\hline Total & $\mathbf{1 2}$ & $\mathbf{1 0 0}$ & $\mathbf{1 3}$ & $\mathbf{1 0 0}$ & $\mathbf{1 3}$ & $\mathbf{1 0 0}$ & $\mathbf{3 8}$ & $\mathbf{1 0 0}$ \\
\hline $\begin{array}{l}\text { Se sim, qual efeito sistêmico? } \\
\begin{array}{l}\text { Melhora no controle } \\
\text { metabólico }\end{array}\end{array}$ & 3 & 25 & 6 & 46,15 & 4 & 30,76 & 13 & 34,21 \\
$\begin{array}{l}\text { Diminuição dos níveis } \\
\text { glicêmicos }\end{array}$ & 2 & 16,66 & 2 & 15,38 & - & - & 4 & 10,52 \\
$\begin{array}{l}\text { Outros } \\
\text { Não respondeu }\end{array}$ & 1 & 8,33 & 1 & 7,69 & 1 & 7,69 & 3 & 7,89 \\
\hline Total & 6 & 50 & 4 & 30,76 & 8 & 61,53 & 18 & 47,36 \\
\hline
\end{tabular}

Você sabia que a Doença Periodontal é considerada a 6a complicação do diabetes?

\begin{tabular}{lcccccccc} 
Sim & 1 & 8,33 & 5 & 38,46 & 2 & 15,38 & 8 & 21,05 \\
Não & 11 & 91,66 & 8 & 61,53 & 11 & 84,61 & 30 & 78,94 \\
\hline Total & $\mathbf{1 2}$ & $\mathbf{1 0 0}$ & $\mathbf{1 3}$ & $\mathbf{1 0 0}$ & $\mathbf{1 3}$ & $\mathbf{1 0 0}$ & $\mathbf{3 8}$ & $\mathbf{1 0 0}$ \\
\hline
\end{tabular}

Você já encaminhou algum paciente diabético para consulta odontológica?

\begin{tabular}{lcccccc} 
Sim & 1 & 8,33 & 3 & 23,07 & 4 & 16 \\
Não & 11 & 91,66 & 10 & 76,92 & 21 & 84 \\
\hline Total & $\mathbf{1 2}$ & $\mathbf{1 0 0}$ & $\mathbf{1 3}$ & $\mathbf{1 0 0}$ & $\mathbf{2 5}$ & $\mathbf{1 0 0}$ \\
\hline
\end{tabular}

Você solicita de forma rotineira o teste de glicemia rápida em diabéticos previamente a procedimentos odontológicos?

\begin{tabular}{lcccc} 
Sim & 11 & 84,61 & 11 & 84,61 \\
Não & 2 & 15,38 & 2 & 15,38 \\
\hline Total & $\mathbf{1 3}$ & $\mathbf{1 0 0}$ & $\mathbf{1 3}$ & $\mathbf{1 0 0}$ \\
\hline
\end{tabular}




\section{DISCUSSÃO}

Estudos recentes afirmam que o DM pode interferir na patogênese da DP, agilizando o seu aparecimento e aumentando sua taxa de gravidade ${ }^{11,14}$. O DM é a alteração do sistema endócrino mais associada negativamente aos quadros inflamatórios/infecciosos da gengiva ${ }^{15}$. Já a DP não tratada pode induzir um estado crônico de resistência à insulina, contribuindo para a hiperglicemia ${ }^{8}$. Tendo em vista que a relação entre essas duas condições ocorre de forma bidirecional, fica evidente a necessidade do acompanhamento multi e interdisciplinar do paciente diabético no âmbito da Atenção Básica, sendo esta a porta de entrada mais usual ao Sistema de Saúde. O presente estudo pôde observar que a maioria dos profissionais atuantes na ESF em Arcoverde/PE são da faixa etária de 31 a 40 anos (19/50\%), predominantemente do sexo feminino (20/53\%) e com tempo de atuação profissional entre 6 e 20 anos (23/60\%).

Todos os profissionais participantes afirmaram já ter atendido um paciente diabético, o que é de concordância com a literatura ${ }^{13,16}$, pois em ambos os estudos com as mesmas categorias profissionais, quase $100 \%$ também afirmaram já ter atendido um paciente diabético. Isso se justifica pelo fato de que a USFs possui um programa voltado para a saúde dos diabéticos, o HiperDia ${ }^{13}$; tal programa do Ministério da Saúde é voltado à prestação da atenção à saúde de modo mais sistematizado e concentrado na USFs, não somente ao diabético, como também ao paciente hipertenso. Um percentual de $68 \%$ dos participantes afirmou conhecer a porcentagem estimada de diabéticos no território adstrito de atuação, o que é um bom número, entretanto, ainda $32 \%$ desconhecem esse dado tão relevante, que serve de embasamento para o planejamento adequado das ações em saúde.

É importante ressaltar que, tanto o médico, como o enfermeiro e cirurgião-dentista devem estar atentos e questionar sempre na anamnese questões referentes à poliúria, polifagia, polidipsia e perda de peso, com o intuito de diagnosticar um possível diabético ${ }^{17}$. Dentre os participantes, $100 \%$ afirmam questionar fatores relacionados ao DM na anamnese. Já $95 \%$ afirmaram que alguma vez suspeitaram durante a anamnese que um paciente poderia ser diabético e solicitaram exames complementares para confirmar essa condição. Dentre os exames mais solicitados pelos profissionais para diagnóstico estão: Glicemia em Jejum (84\%), Hemoglobina Glicosilada (63\%) e Glicemia rápida (55\%). Sobre a hemoglobina glisosilada (HbA1c), é válido ressaltar que, atualmente, o seu valor tem direta correlação com o grau da taxa de progressão da periodontite em pacientes com DM, classificando-os como de progressão moderada $(\mathrm{HbA} 1 \mathrm{c}<7 \%)$ ou rápida $(\mathrm{HbA1c}>7 \%)$ de suas periodontites ${ }^{18}$.

Sobre existir um cuidado especial ao tratar um paciente diabético, $95 \%$ dos participantes consentem com tal afirmativa. De forma geral, a literatura afirma que os portadores de DM bem controlados e sem muitas complicações não necessitam de cuidados especiais. Entretanto, aqueles descompensados apresentam um maior risco de desenvolver infecções, sendo até necessária a prescrição de profilaxia antibiótica previamente a procedimentos cirúrgicos ${ }^{19}$

Quando os profissionais foram questionados sobre conhecer o que é a doença periodontal, 33\% dos médicos e $62 \%$ dos enfermeiros responderam que não. Já $100 \%$ dos CDs afirmaram saber do que se trata, o que é um dado esperado, tendo em vista que a DP é alvo de muitos estudos na Odontologia. Já sobre saber qual o principal sinal clínico da doença periodontal, $58 \%$ dos médicos e $77 \%$ dos enfermeiros o desconhecem.

Aos que afirmaram conhecer o principal sinal clínico da DP (42\% dos médicos, $100 \%$ dos CDs e $23 \%$ dos Enfermeiros), foi perguntado por meio de uma questão subjetiva qual seria esse sinal: apenas $25 \%$ dos médicos, $46 \%$ dos CDs e $15 \%$ dos Enfermeiros elencaram o sangramento em suas respostas; já 38\% dos CDs citaram Bolsa Periodontal ou expressões que a remetem e os demais (15\%) citaram sinais que não se adequam em nenhuma das categorias citadas. Ou seja, de um total de 38 profissionais participantes, 21 afirmaram saber qual o principal sinal clínico da DP, porém apenas 11 profissionais elencaram o sangramento como este sinal. A literatura afirma que o sangramento é sinal mais característico de presença da $\mathrm{DP}^{20}$, todavia, é importante entender que a doença periodontal pode se manifestar por dois quadros clínicos: a gengivite e a periodontite ${ }^{20}$.

Indivíduos que manifestam apenas o quadro de gengivite, já possuem a DP instalada em sua fase incipiente causando alterações no periodonto de proteção. Sendo assim, é importante reconhecer o sangramento gengival como sinal clínico para diagnóstico em estágio inicial da doença, atualmente sob a necessidade de ser observado em pelo menos $10 \%$ dos sítios sondados ${ }^{10}$.

Quando os participantes foram questionados se 0 tratamento periodontal auxilia no controle da glicemia, $60 \%$ responderam "sim", 3\% "não" e 37\% "não sei". Reconhecidamente, a progressão da doença periodontal é favorecida pela falta de controle glicêmico ${ }^{12}$. Já acerca de qual o efeito sistêmico que o tratamento periodontal pode proporcionar ao paciente diabético descompensado com periodontite, apenas $52 \%$ disseram que conhecem este mecanismo, apesar de poder existir resposta inexpressiva ao tratamento periodontal nesses pacientes descompensados, com mais complicações pós-operatórias ${ }^{21}$.

Por meio de uma questão objetiva sobre qual seria o efeito sistêmico, 34\% responderam "melhora no controle metabólico", 10\% "diminuição nos níveis glicêmicos" e 7\% "outros". O restante não respondeu. Afecções odontológicas, como a DP, em pacientes com DM, podem agravar o controle metabólico ${ }^{11,19,22}$. E como qualquer outro tipo de infecção, a doença periodontal pode tornar difícil o controle da glicemia em um paciente diabético, devido ao fato de que a infecção predispõe à resistência à insulina, podendo causar um quadro de hiperglicemia crônica ${ }^{11,22,23}$, o que induz a formação e acúmulo de produtos finais da glicação avançada, que acarreta no aumento do número de periodontopatógenos e altera as funções das células de defesa ${ }^{11,23}$, resultando em uma maior 
destruição tecidual e na diminuição do potencial de reparo do organismo $^{14,24}$

Pacientes com diabetes têm maior probabilidade de desenvolver DP e, neles, esta se instala de forma mais rápida e grave $e^{7,11}$ por prejudicar a função imune celular, diminuir a síntese e renovação de colágeno e induzir a reabsorção óssea alveolar ${ }^{11}$. A literatura considera a DP como a sexta complicação crônica do diabetes ${ }^{25-27}$. Quando os profissionais foram questionados sobre o conhecimento dessa afirmativa, $78 \%$ dos profissionais afirmaram que a desconheciam.

Pacientes com diagnóstico de DM devem ter sua glicemia capilar aferida antes de intervenções odontológicas, com o objetivo de evitar complicações como hipoglicemia ou hiperglicemia ${ }^{27}$. Neste estudo, 85\% dos CDs responderam que "sim"ao questionamento sobre a conduta de solicitaroexame de glicemia rápida de forma rotineira aos pacientes portadores de DM previamente a procedimentos odontológicos. Entretanto, esse seria um dado do qual se esperava unanimidade nas respostas (positivamente), tendo em vista o protocolo definido pelo Ministério da Saúde ${ }^{28}$ (Figura 3) e o elevado risco que é realizar intervenções em pacientes com alterações glicêmicas exacerbadas. Ressaltasse que, normalmente, encontram-se glicosímetros e tiras reagentes nas unidades com USFs do município em que a presente pesquisa foi realizada.

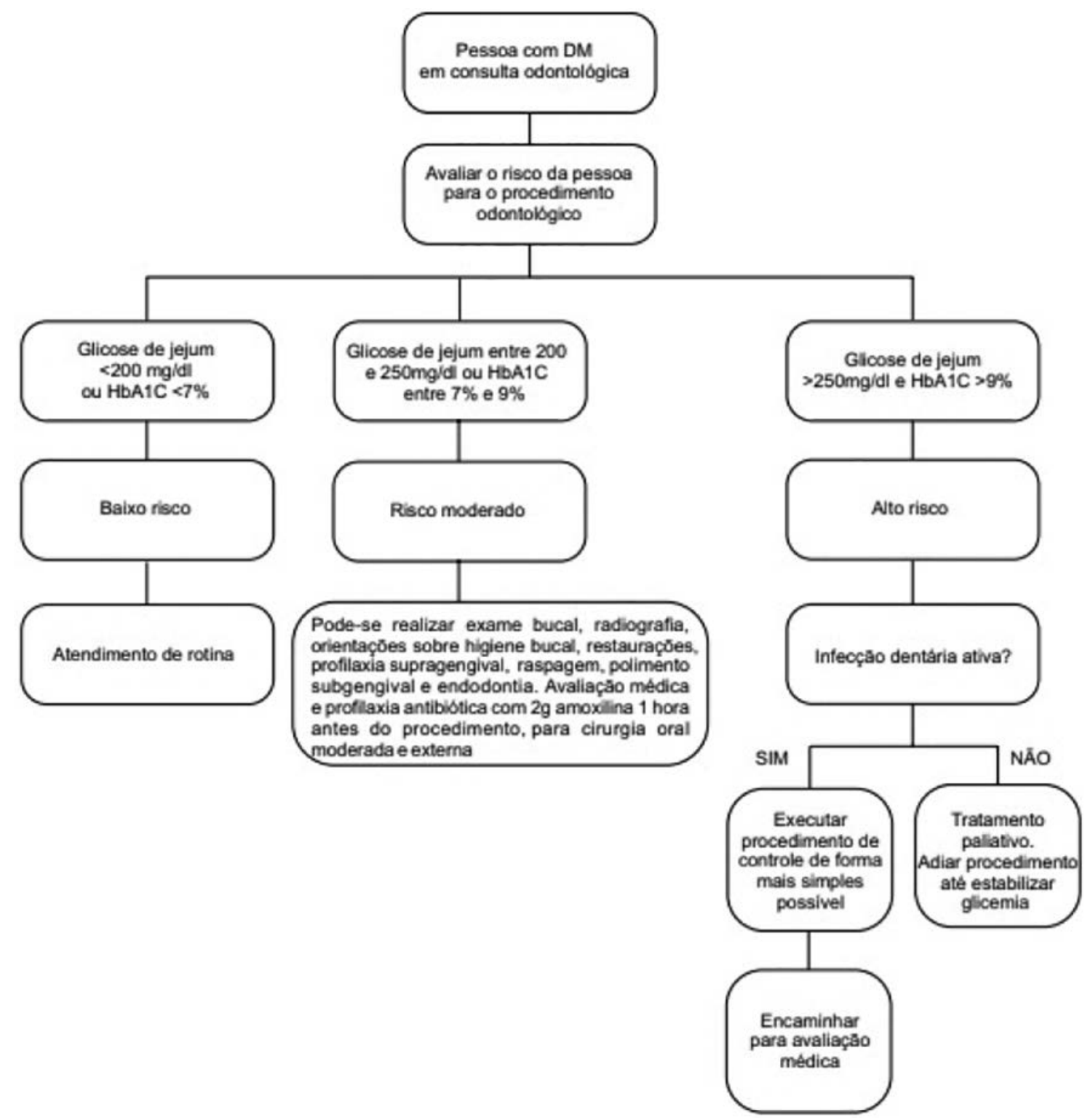

Fonte: Ministério da Saúde, Departamento de Atenção Básica (2013).

Figura 3 - Orientação para manejo clínico de pessoas com DM em consulta odontológica. 
Segundo a orientação definida pelo Departamento de Atenção Básica ${ }^{28}$ para o manejo clínico de pessoas com DM em consulta odontológica, os procedimentos possíveis de serem realizados são definidos mediante o risco (baixo, moderado e alto) desses pacientes e esse risco é definido pela medição da glicose em jejum. Portanto, faz-se imprescindível o amplo acesso ao exame de glicemia na atenção primária, bem como o trabalho multiprofissional dos pacientes cadastrados no "Hiperdia". Autores ressaltaram em seu estudo que o compromisso da equipe multiprofissional sobre a resolutibilidade decorre da relação entre os trabalhadores nessa atenção. Dessa forma, um planejamento integrado focado no problema do usuário melhoraria a atenção prestada e o seguimento dos protocolos propostos entre as diferentes áreas ${ }^{29}$.

Nesse sentido, os usuários pré-diabéticos e diabéticos devem ser encaminhados para avaliação e cuidados odontológicos para que o CD possa atuar em conjunto com toda a equipe na assistência integral à saúde dos pacientes ${ }^{6,19,28}$ Médicos e enfermeiros, em especial, devem estar preparados para reconhecer a doença periodontal consequente da inflamação crônica dos tecidos periodontais que é muito frequente nas pessoas com DM que ainda conservam dentes próprios ${ }^{28}$. Fo questionado apenas para os médicos e enfermeiros se eles já haviam encaminhado algum paciente diabético para consulta odontológica, dos quais $84 \%$ responderam que "não". Estudos têm mostrado que poucos são os indivíduos com Diabetes mellitus que recebem tratamento odontológico na atenção básica à saúde e a busca ativa dos pacientes diabéticos pelo cirurgião-dentista é mínima quando comparada a importância do tratamento periodontal no controle do diabetes ${ }^{8}$.

\section{CONCLUSÃO}

De maneira geral, a maior parte dos profissionais participantes afirmou investigar durante a anamnese questões relacionadas ao diabetes e conhecer os principais exames laboratoriais necessários para o seu diagnóstico. Entretanto, sobre as principais características da doença periodontal, o conhecimento é um tanto fragmentado. No que se refere ao entendimento da relação entre esses dois agravos, pode-se perceber em todas as categorias profissionais escassez de conhecimento sobre o assunto.

Estudos que avaliem a integralidade entre os participantes atuantes nos serviços de saúde são necessários. Sugere-se que a comunidade acadêmica local realize ações de extensão com os profissionais, voltadas ao assunto e comunique à Secretaria de Saúde os principais resultados desta pesquisa; de forma a frisar a importância dessa relação de via bidirecional que necessita de tratamento multi e interdisciplinar.

\section{REFERÊNCIAS}

1. Felipe EM, Chomyszyn-Gajeswska M, Fischer RG. Efeito do tratamento periodontal em pacientes com diabetes mellitus tipo 2. Rev Hosp Univ Pedro Ernesto. 2013;12(1):84-91.
2. Ministério da Saúde, Secretaria de Atenção à Saúde. Cadernos de Atenção Básica, N 17. Brasília: Ministério da Saúde, 2008.

3. Rodrigues TQ, Silva RVC, Ribeiro FS, Pontes AEF. Conhecimento sobre saúde periodontal dos pacientes submetidos à triagem nos postos de saúde de Barretos. Braz J Periodontol. 2014;24(2):19-23.

4. Balejo RDP, Porto S, Cortelli, SC. Bacteriemia em pacientes periodontais: revisão de literatura. Braz J Periodontol. 2014;24(4):29-40.

5. Ministério da Saúde. Projeto SBBrasil 2010: Pesquisa Nacional de Saúde Bucal - Resultados Principais. Brasília: Ministério da Saúde, 2011.

6. Newman MG, Takei HH, Klokkevold PR, Carranza FA. Carranza: Periodontia clínica. 11 ed. Rio de Janeiro: Elsevier, 2012.

7. Rodrigues IL, Silva KG, Sousa RL, Ribeiro RA, Rodrigues RQF, Sousa JNL. Efeito do tratamento periodontal não cirúrgico no nível de hemoglobina glicada e pacientes portadores de diabetes tipo 2 com periodontite crônica: uma revisão sistemática de literatura. Braz J Periodontol. 2015;25(1):47-56.

8. Sousa JNL, Nóbrega DRM, Araki AT. Perfil e percepção de diabéticos sobre a relação entre diabetes e doença periodontal. Rev Odontol UNESP. 2014;43(4):265-72.

9. Abreu IS, Takahashi DY, Silva HAB, Fukushimal LMBA, Andrade PVC, Possamail SMB, et al. Diabetes Mellitus: what periodontists must know. Braz J Periodontol. 2014;24(4):22-8.

10. Caton JG, Armitage G, Berglundh T, Chapple ILC, Jepsen S, Kornman KS, Mealey BL, Papapanou PN, Sanz M, Tonetti MS. A new classification scheme for periodontal and peri-implant diseases and conditions - Introduction and key changes from the 1999 classification. Journal Of Clinical Periodontology. 2018;45(20):1-8

11. Sociedade Brasileira de Diabetes. Diabetes e doença periodontal. Diretrizes da Sociedade Brasileira de Diabetes (2015-2016). São Paulo: A. C. Farmacêutica, 2016.

12. Trentin MS, Carli JP, Ferreira MC, Gambin DJ, Silva SO, Lisboa HRK. Prevalência e severidade da doença periodontal em pacientes portadores de diabetes mellitus tipo 2: um estudo transversal. Biosci J. 2018;34(4):1114-23.

13. Santos IC, Germano SCF, Braz MCA, Araki AT, Santos-Pereira AS, Saba-Chujfi E. Conhecimento dos profissionais da estratégia de saúde da família sobre diabetes e doença periodontal. ImplantNewsPerio. 2016;1(6):1179-87.

14. Vanderlei $A C Q$, Vanderlei JMTMM, Rangel MLR, Silva CAM, Aguiar JP, Carvalho AKFAC. Considerações acerca dos mecanismos patogênicos da doença periodontal. Rev Camp Sab. 2018;4(5):159-73.

15. Tolentino PHMP, Prado MM, Trindade DB, Franco A, Silva RF. A importância da participação do paciente para a manutenção da saúde periodontal - revisão de literatura. Rev Bras Odontol Leg. 2018;5(3):62-73.

16. Souza RRC. Doença periodontal e diabetes: percepção e assistência dos profissionais da estratégia de saúde da família [dissertação]. Patos: Faculdades Integradas de Patos, 2013. 
17. Souza RR, Castro RD, Monteiro CH, Silva SC, Nunes AB. O paciente odontológico portador de diabetes mellitus. Pesq Bras Odontopediatr Clin Integr. 2003;3(2):71-7.

18. Costa R, Resende M, Pinto M, Mendes L. Diagnóstico periodontal: um fluxograma de decisão para a nova classificação. Revista Portuguesa de Estomatologia, Medicina Dentária e Cirurgia Maxilofacial. 2019;60(4):189-96.

19. Terra BG, Goulart RR, Bavaresco CS. O cuidado odontológico do paciente portador de diabetes mellitus tipo 1 e 2 na atenção primária à saúde. Rev APS. 2011;14(2):149-61.

20. Marin C, Holderied FS, Salvati G, Bottan ER. Nível de informação sobre doenças periodontais dos pacientes em tratamento em uma clínica universitária de periodontia. Rev Salusvita. 2012;31(1):19-28.

21. Tsogny-Tsague NF, Lontchi-Yimagou E, Nana ARN, Tankeu AT, Katte JC, Dehayem MY, Bengondo CM, Sobngwi E. Effects of nonsurgical periodontal treatment glycated haemoglobin on type 2 diabetes patients (PARODIA 1 STUDY): a randomized controlled trial in a sub-Saharan Africa population. BMC Oral Health. 2018;18(1):28.

22. Izu AM, Moreira K, Nascimento MCB, Junior RP. Diabetes e a relação com a doença periodontal. Rev Ceciliana. 2010;2(2):23-5

23. Chapple ILC, Genco R. Diabetes and periodontal diseases: consensus report of the joint EFP/AAP workshop on periodontitis and systemic diseases. J. Periodontol. 2013;84:106-12.

24. Janket SJ, Wightman A, Baird AE, Van Dyke TE, Jones JA. Does periodontal treatment improve glycemic control in diabetic patients? A meta-analysis of intervention studies. J Dent Res. 2005;84(12):1154-9.

25. Loe H. Periodontal disease. The sixth complication of diabetes mellitus. Diabetes Care. 1993;16(1):329-34.

26. Almeida RF, Pinho MM, Lima C, Faria I, Santos P, Bordalo C. Associação entre doença periodontal e patologias sistémicas. Rev Port Clin Geral. 2006;22(3):379-90

27. Alves C, Brandão M, Andion J, Menezes R, Carvalho F. Atendimento Odontológico do Paciente com Diabetes Melito: Recomendações para a Prática Clínica. Rev Ci Med Bio. 2006;5(2):97-110.

28. Ministério da Saúde, Secretaria de Atenção à Saúde, Departamento de Atenção Básica. Estratégias para o cuidado da pessoa com doença crônica: diabetes mellitus - Cadernos de Atenção Básica, N 36. Brasília: Ministério da Saúde, 2013.

29. Lopes AS, Vilar RLA, Melo RHV, França RCS. O acolhimento na Atenção Básica em saúde: relações de reciprocidade entre trabalhadores e usuários. Saúde em Debate. 2015;39(104):114-23. 